\title{
Pionic dressing of baryons in chiral quark models
}

\author{
Alexander N. Kvinikhidze*, Michael C. Birse \\ Theoretical Physics Group, Department of Physics and Astronomy, University of Manchester, \\ Manchester M13 9PL, United Kingdom \\ Boris Blankleider \\ Department of Physics, The Flinders University of South Australia, Bedford Park, SA 5042, \\ Australia
}

(October 30, 2018)

\begin{abstract}
We present a method for constructing the complete set of meson-exchange corrections to baryon observables described by covariant chiral quark models. The meson corrections are expressed in terms of an unperturbed valencequark Green's function. This method is illustrated for the case of an NJL model. It also enables us to discuss, in terms of approximations used for this unperturbed Green's function, the treatments of meson corrections in two other chiral quark models.
\end{abstract}

\section{INTRODUCTION}

Chiral symmetry is one of the most important features of QCD at low energies. This approximate symmetry is spontaneously broken by the quark condensate, leading to constituent masses for the quarks and to pions which are, to a good approximation, the corresponding Goldstone bosons. As a result pions are much lighter than all other mesons and so the cloud of virtual pions surrounding a baryon can make significant contributions to the properties of that baryon. Any realistic model for the structure of baryons should therefore take account of their pion clouds.

As approximate Goldstone bosons, pions with low momenta interact relatively weakly with other hadrons. This forms the basis for chiral perturbation theory (ChPT), in which hadron properties and scattering amplitudes are expanded in powers of momenta and the pion mass (for a review, see [1]). It also provides a motivation for treating the pion cloud perturbatively in chiral quark models. Such a treatment ensures that at least the longestrange effects of the pion cloud are described correctly, since these involve virtual pions with

* On leave from Mathematical Institute of Georgian Academy of Sciences, Tbilisi, Georgia. 
low momenta. These effects can be checked by comparing contributions to hadron properties that depend nonanalytically on the square of the pion mass with the model-independent predictions of ChPT.

A range of chiral quark models for baryon structure can be found in the literature. These include chiral or "cloudy" bag and soliton models [2,3] and chiral versions of constituent quark models [1] 7]. In all of these, pions are introduced as elementary degrees of freedom and in many cases their contributions are calculated perturbatively.

In contrast, models of the Nambu-Jona-Lasinio (NJL) type are based on relativistic quarks only, and the quark condensate and pions are generated by the interactions between these quarks (for a review, see: [8]). As such they represent a step closer to QCD, although problems with implementing confinement remain to be solved satisfactorily. In these models baryon states have been constructed by solving the relativistic Faddeev equation [9 11], but this approach does not include the pion cloud. The part of the cloud corresponding to the pairwise meson exchange between quarks has been studied in Ref. [15]. Recently a covariant version of the treatment used in the cloudy-bag model has been applied to calculate pion cloud effects based on a Faddeev approach to the nucleon [16,17. Also, a quite different, noncovariant approach to the calculation of pion cloud effects in models with composite pions has been proposed by Bicudo et al. [18.

In the present work we address the problem of mesonic corrections within an NJL model, deriving covariant expressions for the complete set of one-loop corrections to baryon masses and couplings to currents. The approach is based on the "gauging of equations" method [19 for constructing photon couplings to strongly interacting systems of particles. Our approach is applicable to any set of one-boson contributions to such systems and was recently used to construct a complete set of electromagnetic corrections [20. As has been noted previously in the context of NJL models for mesons [21 24], such completeness is important since it ensures that physical quantities calculated in this approach satisfy the relevant symmetry constraints. The general features of the approach also allow us to comment on the approximations used in cloudy-bag and chiral quark models, which have recently been the subject of some debate 25 28].

\section{COVARIANT MESONIC CORRECTIONS}

A method has recently been presented for calculating all possible electromagnetic corrections, at order $e^{2}$, to any quark or hadronic model whose strong interactions can be represented nonperturbatively by integral equations [20]. We will use this method to calculate in a covariant manner all mesonic corrections to a relativistic quark model. For this

\footnotetext{
${ }^{1}$ We should mention that a completely different approach to baryons in these models uses a meanfield treatment where baryons are described as Skyrmion-like solitons [12,13]. This is expected to be valid in the limit of a large number of colours. However in the realistic case of three colours the Faddeev approach is likely to be more appropriate. An attempt to hybridise the two approaches can be found in Ref. [14.
} 
purpose all we need to do is to summarise the main model-independent results of Ref. [20], applied to exchange of mesons rather than photons.

In such a model, the Green's function $G$ describing a bare system of quarks satisfies an integral equation whose symbolic form is

$$
G=G_{0}+G_{0} K G,
$$

where $G_{0}$ is the "free" Green's function. Note that this need not describe the propagation of three free quarks. In a Faddeev approach, for instance, it is the Green's function for one free quark and an interacting pair of quarks (often referred to as a diquark, although the pair need not be bound).

The lowest-order mesonic correction to $G$ is denoted by $\delta G$. This should contain all possible insertions of the meson propagator, $D_{\mu \nu}$. In chiral quark models with elementary mesons this is just a standard boson propagator, but in NJL-type models it is essentially the quark-antiquark $T$-matrix, as discussed below. A four-vector-like notation is used for the indices $\mu$ and $\nu$ which label the meson channels. This will allow for applications to models with vector or axial mesons. The propagator is diagonal in its indices, except for the mixing between pseudoscalar and axial channels which occurs in models with spin-1 mesons. The set of corrections includes mesonic insertions within the kernel $K$ which do not start or finish on an external quark line. These provide a contribution $\delta K$ to the kernel. Similarly insertions within the free Green's function $G_{0}$ provide a contribution $\delta G_{0}$.

All contributions with a meson which connects two elements of the integral equation Eq. (11) are constructed by the "gauging of equations" method [19] which in this case attaches an external meson in all possible ways to each of the unperturbed quantities $G_{0}, K$ and $G$. The corresponding vertices are given by

$$
\Gamma_{0}^{\mu}=G_{0}^{-1} G_{0}^{\mu} G_{0}^{-1}, \quad \Gamma^{\mu}=\Gamma_{0}^{\mu}+K^{\mu}, \quad G^{\mu}=G \Gamma^{\mu} G .
$$

An equation for the complete set of lowest-order mesonic corrections to $G$, denoted by $\delta G$, then follows from Eq. (四):

$$
\delta G=\delta G_{0}+\delta G_{0} K G+G_{0} \delta K G+G_{0} K \delta G+\left(G_{0}^{\mu} K^{\nu} G+G_{0}^{\mu} K G^{\nu}+G_{0} K^{\mu} G^{\nu}\right) D_{\mu \nu} .
$$

Writing

$$
\delta G=G \Delta G
$$

this can be formally solved to give

$$
\Delta=\delta K+G_{0}^{-1} \delta G_{0} G_{0}^{-1}+\left(\Gamma^{\mu} G \Gamma^{\nu}-\Gamma_{0}^{\mu} G_{0} \Gamma_{0}^{\nu}\right) D_{\mu \nu} .
$$

The quantity $\Delta$ consists of the complete set of one-loop mesonic corrections to the unperturbed kernel $K$. The third term on the right hand side (RHS) contains the physical intermediate states of three quarks plus a meson. There are significant cancellations between the second and fourth terms, leaving contributions which correct the $G_{0}$ piece of the third term for omissions and overcounting. Note that the contributions of these terms include disconnected pieces where the particles in $G_{0}$ are individually dressed by meson loops. These can be easily recast as corrections to the "free" propagator $G_{0}$. 
Although our main focus here is on covariant approaches, it is worth noting that the same formalism also applies to time-ordered perturbation theory. The only difference is that the Green's functions involved are functions of three-momenta rather than four-momenta. A method for applying time-ordered perturbation theory to mesonic corrections in models of this type has recently been outlined in Ref. [18], although that work proposed truncating the sum over intermediate baryon states to just the nucleon and $\Delta$. However, within a time-ordered approach, it is difficult to ensure that vertex functions satisfy the constraints of Lorentz invariance. This is necessary if one wants to calculate processes involving finite momentum transfer to a baryon, or to treat correctly recoil of the intermediate baryons in meson-loop diagrams. In addition, a time-ordered approach would make it difficult to include quark "z-diagrams", since these would go beyond a basis of states with three valence quarks (plus mesons). Such effects are expected to be important in models where the quarks have strong couplings to scalar mesons and couplings to pions with a pseudoscalar form. It is also difficult to regularise time-ordered approaches to NJL models in a way that respects Lorentz invariance. For these reasons we have concentrated here on developing a covariant treatment.

\section{NJL MODEL FOR THE NUCLEON}

The NJL model [8] is a relativistic quark model which reflects many aspects of the spontaneously broken chiral symmetry of QCD. It therefore provides a useful framework within which to investigate the role of mesonic corrections.

The simplest version of the model is defined in terms of an isospin-doublet colour-triplet quark field $\psi$ and has the Lagrangian density

$$
\mathcal{L}=\bar{\psi}\left(i \not \partial-m_{0}\right) \psi+g_{\pi}\left[(\bar{\psi} \psi)^{2}-\left(\bar{\psi} \gamma_{5} \boldsymbol{\tau} \psi\right)^{2}\right]
$$

where $\boldsymbol{\tau}$ is the vector of isospin Pauli matrices, and $m_{0}$ is the current quark mass (which explicitly breaks chiral symmetry).

Chiral symmetry is spontaneously broken in this model by condensation of quarkantiquark pairs. These generate a constituent mass for the quarks, which can be found by solving the Schwinger-Dyson (SD) equation for the quark propagator

$$
S(p)=S_{0}(p)+S_{0}(p) \Sigma(p) S(p)
$$

where $S_{0}(p)$ is the bare quark propagator and the self-energy $\Sigma$ is, in the Hartree approximation,

$$
\Sigma(p)=-i g_{\pi} \int \frac{d^{4} k}{(2 \pi)^{4}} \operatorname{tr}[S(k)] .
$$

Here "tr" denotes a trace over Dirac, colour and flavour indices. The integral over fourmomenta is, of course, divergent and so needs to be regularised. This may be done either by using one of the various cut-off schemes which can be found in the literature, or by introducing form-factors to smear out the local interaction. Provided that the coupling $g_{\pi}$ is large enough, this SDE has a nonzero solution for $\Sigma$. 
The quark-antiquark $T$-matrix can be found by solving a Bethe-Salpeter (BS) equation, which in the random-phase approximation is a simple algebraic equation. For example, in the pion channel this yields

$$
D_{\pi}(p)=\frac{4 i g_{\pi}}{1-2 i g_{\pi} \Pi_{\pi}\left(p^{2}\right)},
$$

where the basic quark-antiquark loop integral is

$$
\Pi_{\pi}\left(p^{2}\right) \delta_{i j}=\int \frac{d^{4} k}{(2 \pi)^{4}} \operatorname{tr}\left[i \gamma_{5} \tau_{i} S(p+k) i \gamma_{5} \tau_{j} S(k)\right] .
$$

In the simplest NJL model there is a similar $T$-matrix in the scalar isoscalar ( $\sigma$-meson) channel. In more general versions of the model, interactions in the vector and axial-vector channels are treated in the same way.

In the treatment of the model considered here, the nucleon is described by a three-quark vertex function which satisfies a four-dimensional Faddeev equation. The local nature of the quark-quark interaction means that the structure of this integral equation is similar to that of a BS equation. At lowest order the kernel $K$ can be pictured as exchange of a quark between a quark and two interacting quarks (a "diquark").

The coupling strengths of the quark-quark interaction can be found by Fierz-transforming it into these channels [10]. The colour- $\overline{3}$ diquark channels are the relevant ones for formation of a nucleon. For a local interaction, there are two such channels: scalar isoscalar and axialvector isovector. The transformed interaction Lagrangian has the form

$$
\mathcal{L}_{I}=g_{S} \sum_{a}\left(\bar{\psi} \gamma_{5} C \tau_{2} \beta^{a} \bar{\psi}^{T}\right)\left(\psi^{T} C^{-1} \gamma_{5} \tau_{2} \beta^{a} \psi\right)+g_{A} \sum_{i, a}\left(\bar{\psi} \gamma_{\mu} C \tau_{i} \tau_{2} \beta^{a} \bar{\psi}^{T}\right)\left(\psi^{T} C^{-1} \gamma^{\mu} \tau_{2} \tau_{i} \beta^{a} \psi\right)
$$

where

$$
\left(\beta^{a}\right)_{i k}=i \sqrt{\frac{3}{2}} \epsilon_{a i k},
$$

and $g_{s}$ and $g_{a}$ are the couplings in these channels. [f For the original NJL model, these couplings can be related to $g_{\pi}$ as described in Ref. [10]. In the scalar-diquark channel, the propagation of two interacting quarks can be described by the $T$-matrix,

$$
D_{s}(p)=\frac{4 i g_{s}}{1-2 i g_{s} \Pi_{s}\left(p^{2}\right)},
$$

where $\Pi_{s}(p)$ has the same form as in the pion channel, Eq. (10). The interaction between two quarks in the axial diquark channel leads to a $T$-matrix whose form is similar to that in a vector-meson channel (see Ref. [10] for more details).

\footnotetext{
${ }^{2}$ Other contributions including colour- 6 diquark channels and the chiral partner of the axial diquark, $\bar{\psi} \gamma_{\mu} \gamma_{5} C \tau_{2} \beta^{a} \bar{\psi}^{T}$, are generated by the Fierz-transformation. We have not written these down since they are not needed for the present discussion.
} 


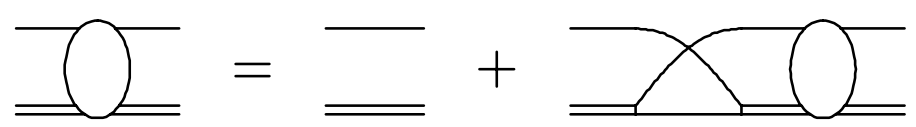

FIG. 1. The Faddeev equation for the quark-diquark Green function.

(a)

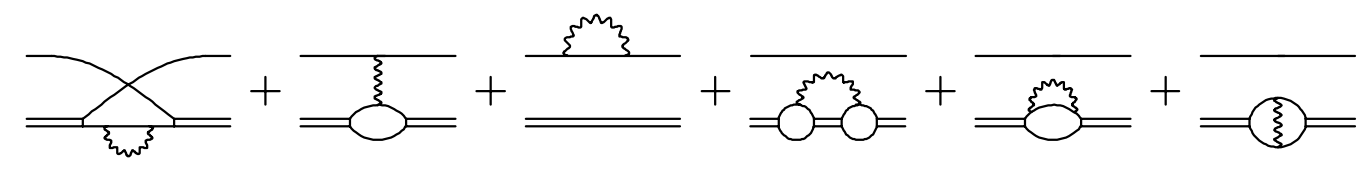

(b)

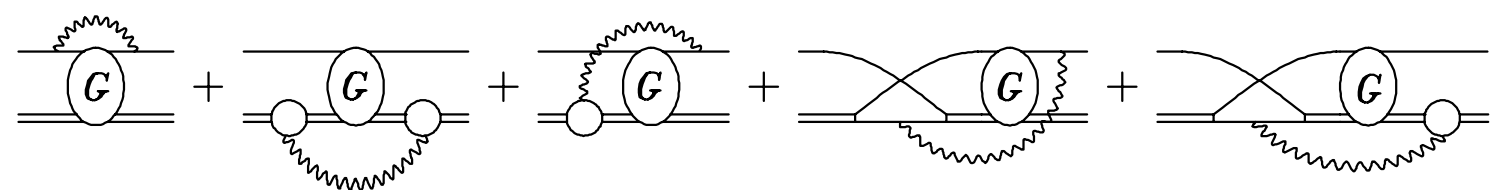

(c)

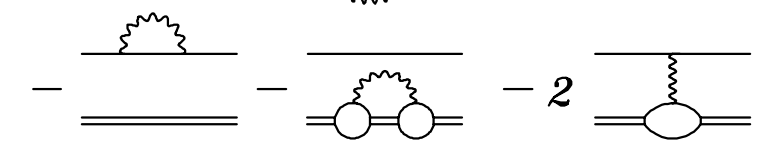

(d)

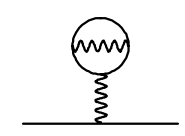

FIG. 2. The mesonic corrections, $\Delta$, to the nucleon in the NJL model. Wavy lines denote meson propagators in the RPA and double lines the corresponding diquark propagators. In line (a) the first diagram is the term $\delta K$ in Eq. (5), and the rest form $G_{0}^{-1} \delta G_{0} G_{0}^{-1}$. The diagrams of line (b), along with the ones formed by swapping initial and final states in the last three diagrams, make up $\Gamma^{\mu} G \Gamma^{\nu} D_{\mu \nu}$. Those of line (c) form the subtraction term $-\Gamma_{0}^{\mu} G_{0} \Gamma_{0}^{\nu} D_{\mu \nu}$. In addition tadpole-type insertions on the quark lines need to be included. For reasons of space, we show only one representative diagram of this class as $(d)$.

The corresponding Faddeev equation is represented diagrammatically in Fig. 1. It can be written in the form of Eq. (四) by defining the "free" quark-diquark propagator

$$
G_{0}(P)=S(P-p) D_{s}(p) .
$$

The quark-diquark interaction kernel is given by the quark exchange term

$$
K_{a^{\prime} a}\left(p^{\prime}, p\right)=\gamma_{5} \beta^{a} S\left(p^{\prime}+p\right) \gamma_{5} \beta^{a^{\prime}} .
$$

When the axial diquark channel is included, the kernel must be extended to a $2 \times 2$ matrix which couples the two spin-isospin channels, as described in Ref. [10].

\section{MESONIC CORRECTIONS TO THE NUCLEON}

The mesonic corrections to this model can be found by applying the general formulation of Eqs. (18)-(5) to the Faddeev equation outlined above. The resulting expression for $\Delta$ is represented diagrammatically in Fig. 2. Mesonic corrections to the nucleon in the NJL model have previously been studied by Ishii [15] but it should be noted that the set of diagrams in that work is incomplete. Only the diagrams of Fig. 3 were included, with subtracted meson propagators as discussed below.

The full set of diagrams includes not only the meson exchanges between the quarks treated by Ishii [15] but also the meson clouds of the quark and diquark. In calculating 

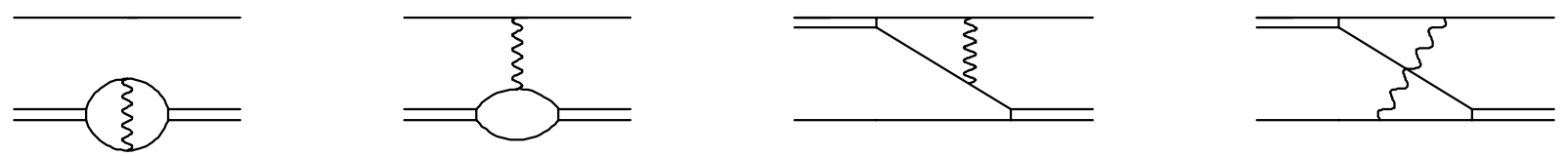

FIG. 3. The parts of the mesonic corrections where the subtracted meson propagator of Eq. (18) should be used.

the pion-cloud contributions it is essential to attach the pion in all possible ways to the dressed quark propagator obtained from the Hartree approximation 21,24. As well as direct dressings of the quark lines, as in Fig. 2(a), this includes attachments within the Hartree self-energy and generates tadpole diagrams like Fig. 2(d) where a pion loop is connected to a quark line via an interacting quark-antiquark pair in the scalar, isoscalar channel. The exchanged quark-antiquark pair is represented in Fig. Z(d) by a sigma-meson propagator. There is a strong cancellation between these diagrams and the basic pion loop attached to a single quark. This cancellation is needed to get the correct leading nonanalytic (LNA) terms in the chiral expansion in any model where the pion-fermion coupling has a pseudoscalar $\left(\gamma_{5}\right)$ form [21]24.

The complete one-meson correction to the nucleon mass in this model can be calculated as the expectation value of $\Delta$ in the lowest-order wave function obtained from the homogeneous equation corresponding to Eq. (11). This can be written

$$
\delta M=\bar{\Psi} \Delta \Psi
$$

where $\Psi$ satisfies

$$
\Psi=G_{0} K \Psi
$$

In models where the mesons are not introduced as elementary fields, some care should be taken with the quark-antiquark T-matrix, Eq. (9). In the mesonic dressing of a single quark or one-loop corrections to meson properties, this $T$-matrix can be used as a meson propagator to generate a complete set of terms at next-to-leading order in $1 / N_{c}$ where $N_{c}$ is the number of colours [23,29,24]. These include terms which are linear in the coupling $g_{\pi}$, such as the exchange or Fock term corresponding to the Hartree self-energy of Eq. (8). For a diquark or a baryon, these linear terms are already included in the interactions between the quarks. Hence meson exchanges between two quarks which do not otherwise interact should be described by the subtracted $T$-matrix

$$
\bar{D}_{\mu \nu}(p)=D_{\mu \nu}(p)-4 i g_{\pi} \delta_{\mu \nu} .
$$

In the case of the pion, this has the form

$$
\bar{D}_{\pi}=\frac{-8 g_{\pi}^{2} \Pi_{\pi}\left(p^{2}\right)}{1-2 i g_{\pi} \Pi_{\pi}\left(p^{2}\right)} .
$$

This subtracted T-matrix should be used in the diagrams of Fig. 3, which form part of Fig. 2.

For a single quark (or a meson) the mesonic one-loop diagrams, including the Fock terms, form the leading corrections to the properties of these particles in a systematic expansion 
in powers of $1 / N_{c}$. In the case of baryons, things are not so clean. Both Hartree and Fock terms contribute to the baryon energy at leading order $\left(N_{c}^{1}\right)$. Meson exchanges between the quarks in a baryon are also of leading order, as are nonlinear terms arising from interactions among the mesons. In the large- $N_{c}$ limit, this provides a justification for treating the baryon as a soliton, as in the Skyrme model. Such treatments have been quite widely applied to NJL-type models [12,13]. They form a radically different approach to baryon structure from the one outlined here and it is still unclear which is the better for the case of interest, $N_{c}=3$. Indeed a hybrid of the two has been proposed in Ref. [14]. Ultimately, numerical calculations of the size of the kernel based on Eq. (18) will indicate whether a perturbative treatment is adequate.

If it is found that the shorter-range pieces of $\bar{D}$ are too strong to be treated perturbatively, then one possible way to improve the convergence of the approach may be to vary the strength of the subtraction term. This must be compensated by adjusting the strength of the interaction used in the bare equation for $G$. That would allow the strong short-range effects to be treated self-consistently in this equation, leaving the longer-range effects to act as a residual interaction.

Note that the loop integral Eq. (10) falls off at high momenta in this type of model. Consequently the subtracted $T$-matrix Eq. (18) also falls off; the enhancements due to the Goldstone-boson nature of the pion only appear at low momenta. It is thus the underlying interaction responsible for spontaneous symmetry breaking which controls the short-range interaction between quarks, not the short-range part of Goldstone-boson-exchange as suggested by some authors [6]. Provided that the quarks in a baryon can be assumed to interact in $s$-waves, only the strengths of the interactions in the scalar and axial diquark channels are relevant.

The inclusion of interactions between the quarks while a meson is in flight, Fig. 2(b), is crucial to ensuring the correct energy denominators for the intermediate baryon-meson states. The binding energy of the nucleon depends on both the scalar and axial diquark couplings, whereas that of the $\Delta$ depends only on the axial one. The short-range interaction thus has an implicit spin-dependence, which splits the bare nucleon and $\Delta$ states. This means that, provided the scalar and axial diquark couplings are chosen to give the correct $\mathrm{N}-\Delta$ splitting, the only intermediate states in the diagrams of Fig. 2(b) with energy denominators of order $m_{\pi}$ are pion-nucleon ones. These will generate the correct LNA terms in a chiral expansion, for example the order- $m_{\pi}^{3}$ term in the nucleon mass [1].

A model of this type can therefore help to resolve some of the issues which have arisen in comparisons of the results of chiral quark models with those of the cloudy bag model [25 28]. In order to get the correct LNA terms (which are a signal that chiral symmetry has been correctly implemented) it is crucial that the full propagator $G$ is used, allowing the quarks to interact while the meson is in flight. This means that the nucleon-plus-pion states necessary for the LNA terms are included, as in the cloudy bag model [2]. However, in contrast to the elementary bag model (i.e. one defined strictly by the cloudy bag model Lagrangian [2]]), in our approach the spin-dependence of the interaction binding the "bare" baryons means that the $\Delta$ is not degenerate with the nucleon and so it does not contribute to the LNA terms in the nucleon mass. Although this problem is avoided in practical bag model calculations where the bare baryon in the baryon-plus-pion state is replaced by a physical one, such an approach is, apparently, equivalent to resumming a subset of mesonic 
corrections to all orders in the Lagrangian-based description. Such a sum may give rise to conflicts with chiral symmetry at higher orders. In a related context, it has been noted that if $\pi N$ loops are resummed in this way then one should also dress the $\pi N$ vertex with pion loops [17].

The chiral quark model of Glozman and coworkers [6,27,30 includes pion loops which dress the quarks individually, effectively treating the quarks as non-interacting while the pion is in flight. In addition, retardation is neglected in the pion exchanges between quarks. These two approximations mean that the current treatments of this model do not generate the correct LNA terms. These would require the inclusion of interactions between the quarks while the meson is in flight, together with the corresponding retardation effects.

Finally it is also worth noting that, in a model of the NJL type, a complete set of intermediate baryon states can be included in the meson-loop diagrams. This will make it possible to examine the role of excited baryons in the mesonic dressing. Although the contributions of individual excited states fall off with energy, there is an infinite number of such states, which may be unbound as well as bound in a nonconfining model. The sum over these states would diverge in the absence of a cut-off. We therefore expect that they could make significant contributions to the mesonic dressing in the approach described here. In this way it will be possible to assess the accuracy of truncating to intermediate nucleon and $\Delta$ states only, as done in the original cloudy bag model [2] and more recently in a model of the type discussed here [16, 17].

In this recent work, Oettel and coworkers [16,17 have calculated pion-loop contributions to nucleon properties within a Faddeev approach, with a covariant version of the approximation used in the cloudy-bag model. In the original cloudy-bag model [2], pion loops were calculated keeping only $\pi N$ and $\pi \Delta$ intermediate states and the baryons were treated as static. In Refs. [16,17 these loops are calculated using fully covariant propagators for the nucleon and $\Delta$ but keeping only the poles corresponding to the lowest-energy solutions to the Faddeev equation in each channel. In esssence, this replaces their relativistic quark model by a model of elementary baryons and mesons. Oettel et al. also take care to include the tadpole diagrams required for the correct chiral behaviour. Their loop integrals are made finite by form factors at the meson-baryon vertices. These form factors can be regarded either as ad hoc regulators of the sort typically used in NJL models or as consequences of the internal structure of the hadrons. Although this approach respects chiral constraints and provides good estimates of the largest individual loop contributions, it does not give the complete loop correction since excited baryon states have been omitted.

\section{MESONIC DRESSING OF CURRENTS}

In order to calculate electromagnetic and weak properties of the nucleon, we need to construct the corresponding currents. At lowest order (no meson loops) the electromagnetic current operator to be used in a three-quark state can be obtained diagramatically by attaching a photon everywhere in Eq. (四). The resulting expression for the matrix element of the current has the form

$$
J^{\mu}=\bar{\Psi}\left(\tilde{\Gamma}_{0}^{\mu}+\tilde{K}^{\mu}\right) \Psi
$$


Here $\tilde{\Gamma}_{0}^{\mu}$ denotes the sum of single-quark and diquark currents, and $\tilde{K}^{\mu}$ the interaction current where the photon is attached to the exchanged quark. This corresponds to the impulse approximation in the three-quark basis, properly subtracted to avoid double counting [31]. The corresponding axial current can be found in the same way by making an axial-vector insertion instead of attaching a photon. Currents like Eq. (20) have been extensively studied in the NJL model [32] as well as in QCD-motivated Faddeev [33] and SD approaches [34. The same methods can also be used to calculate the matrix element of any operator which is bilinear in the quark fields, such as the pion-nucleon sigma commutator.

At the next order, we need to consider the one-loop mesonic contributions to the currents, $\tilde{\Delta}^{\mu}$. These have a similar origin to the contributions to the kernel, $\Delta$, and so can be obtained by attaching a photon everywhere in $\Delta$, including the meson. The attachments to the composite mesons and diquarks are realised by direct couplings to quark currents in the $q \bar{q}$ and $q q$ loops respectively.

In addition we need to take into account the effects of mesonic fluctuations on the bound state wave function. If the full wave function is written as $\Psi+\delta \Psi$, it satisfies the BetheSalpeter-type equation

$$
(\Psi+\delta \Psi)=G_{0}(K+\Delta)(\Psi+\delta \Psi)
$$

The perturbation of the wave function $\delta \Psi$ can be expressed as [35]

$$
\delta \Psi=\left[G_{B} \Delta-\frac{1}{2 M}\left(\bar{\Psi} P^{\mu} \frac{\partial \Delta}{\partial P^{\mu}} \Psi\right)_{|P|=M}\right] \Psi .
$$

In the first term, $G_{B}$ denotes the unperturbed Green function $G$ with the bound-state pole subtracted off. This term incorporates configuration-mixing effects generated by mesonexchange between the quarks. The second term in this expression is just a wave-function renormalisation, analogous to that in the cloudy bag model. It involves the derivative of the meson-exchange part of the kernel with respect to the the total 4-momentum, evaluated at the unperturbed nucleon mass.

The full one-loop contribution to the current matrix element is finally

$$
\delta J^{\mu}=\bar{\Psi} \tilde{\Delta}^{\mu} \Psi+\bar{\Psi}\left(\tilde{\Gamma}_{0}^{\mu}+\tilde{K}^{\mu}\right) \delta \Psi+\delta \bar{\Psi}\left(\tilde{\Gamma}_{0}^{\mu}+\tilde{K}^{\mu}\right) \Psi .
$$

Again, it is important that $\tilde{\Delta}^{\mu}$ contain the full propagator $G$, allowing the quarks to interact while the meson is in flight, see Fig. \$. As in the case of the nucleon mass, this generates the correct LNA terms in chiral expansions of the electromagnetic radii of the nucleon [1]. In particular the isovector charge radius contains a term of order $\ln m_{\pi}$ and the isovector magnetic radius one of order $m_{\pi}^{-1}$. In both cases these LNA terms contribute significantly to the full radii and so any calculation should include pion-nucleon loops with the correct energy denominators.

Neglecting the interactions while the pion is in flight would lead to expressions for the currents like those in the quark model of Glozman and coworkers [6, 30, 36], which do not contain the correct LNA terms. Truncating the sum over intermediate states to the nucleon and $\Delta$ only would lead to an approximation similar to the cloudy-bag model, with the correct LNA terms [26]. 


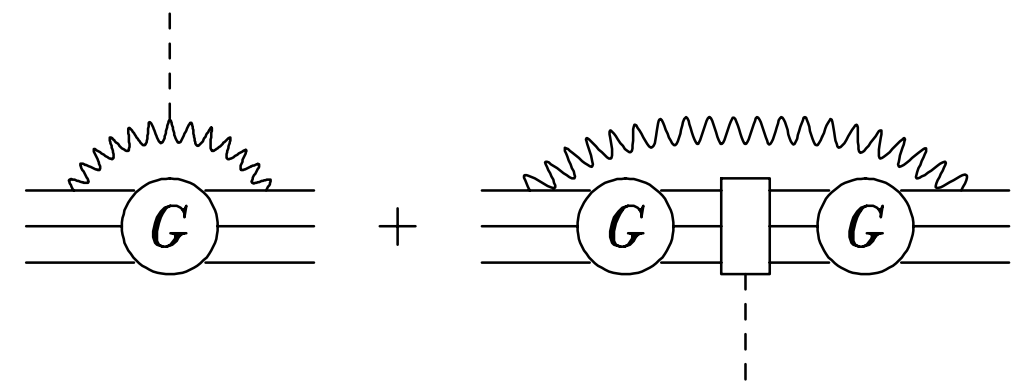

FIG. 4. Mesonic corrections of three-quark (baryon) currents where the quarks interact while the meson is in flight. The dashed line denotes an external photon or other operator.

\section{CONCLUSION}

We have presented a method for constructing a complete set of one-loop mesonic corrections to the nucleon in a chiral quark model, treated in a covariant manner. Our approach is based on the "gauging of equations" method which has previously been used to construct electromagnetic corrections to hadronic systems. It ensures that a complete set of one-loop corrections is generated, with no double counting. This completeness is essential if physical quantities calculated in this approach are to satisfy the constraints of chiral symmetry and gauge invariance.

To illustrate the method, we have discussed in some detail its application to a particular covariant chiral quark model: the NJL model. The method starts from an unperturbed valence-quark Green's function, which in this case is obtained from a Faddeev equation. The complete set of mesonic insertions is used to generate the one-loop contribution to the kernel. The same method can also be used to construct photon-nucleon couplings in a way that respects current conservation.

A central feature of this approach is the use of the unperturbed Green's function for three interacting valence quarks. This means that while a meson is in flight the quarks propagate in physical baryon states, as described at leading order in the meson-loop expansion. The fact that the NJL model gives rise to an $\mathrm{N}-\Delta$ splitting at this order means that the only nonanalytic terms in the chiral expansion are generated by pion-nucleon states. This ensures that the method will give the correct leading nonanalytic terms in nucleon observables, in agreement with ChPT. This is particularly important for electromagnetic radii, where these nonanalytic terms make significant contributions.

The usual treatment of the cloudy-bag model similarly gives the correct leading nonanalytic terms in nucleon observables. However in models of the type discussed here, there is no need to truncate the unperturbed Green's function to just nucleon and $\Delta$ states. A complete set of baryon states can be summed over, regulated by the cut-off which forms an intrinsic part of any NJL-type model.

In contrast, the chiral quark model of Glozman and coworkers effectively uses a free Green's function to describe the propagation of the quarks in meson-baryon intermediate states. It also neglects retardation effects on the exchange of pions between quarks. As a result this model, in the framework used so far, fails to give the correct nonanalytic terms in the chiral expansion. However this could be remedied by a treatment based on the approach 
described here, or the corresponding time-ordered version.

\section{ACKNOWLEDGEMENTS}

This work was supported by the EPSRC. We are grateful to T. Cohen and L. Glozman for helpful discussions. 


\section{REFERENCES}

[1] V. Bernard, N. Kaiser and U.-G. Meissner, Int. J. Mod. Phys. E4 (1995) 193 hepph/9501384].

[2] A. W. Thomas, Adv. Nucl. Phys. 13 (1984) 1.

[3] M. C. Birse, Prog. Part. Nucl. Phys. 25 (1990) 1.

[4] K. Shimizu, Phys. Lett. B148 (1984) 418; D. Robson, Phys. Rev. D35 (1985) 1029; K. Maltman, Nucl. Phys. A446 (1985) 623.

[5] F.Fernandez, A. Valcarce, U. Straub and A. Faessler, J. Phys. G: Nucl. Part. Phys. 19 (1993) 2013.

[6] L. Ya. Glozman and D. O. Riska, Phys. Rep. 268 (1996) 263 hep-ph/9505422.

[7] L. Ya. Glozman, Z. Papp, W. Plessas, K. Varga and R. F. Wagenbrun, Phys. Rev. C57 (1998) 3406 nucl-th/9705011].

[8] S. P. Klevansky, Rev. Mod. Phys. 64 (1992) 649.

[9] A. Buck, R. Alkofer and H. Reinhardt, Phys. Lett. B 286 (1992) 29.

[10] N. Ishii, W. Bentz and K. Yazaki, Phys. Lett. B 318 (1993) 26; Nucl. Phys. A587 (1995) 617.

[11] S. Huang and J. Tjon, Phys. Rev. C 49 (1994) 1702.

[12] R. Alkofer, H. Reinhardt and H. Weigel, Phys. Rep. 265 (1996) 139 hep-ph/9501213.

[13] C. V. Christov et al., Prog. Part. Nucl. Phys. 37 (1996) 91 hep-ph/9604441].

[14] U. Zückert, R. Alkofer, H. Weigel and H. Reinhardt, Phys. Rev. C55 (1997) 2030 nuclth/9609012.

[15] N. Ishii, Phys. Lett. B431 (1998) 1.

[16] M. B. Hecht, M. Oettel, C. D. Roberts, S. M. Schmidt, P. C. Tandy and A. W. Thomas, Phys. Rev. C65 (2002) 055204 nucl-th/0201084.

[17] M. Oettel and A. W. Thomas, nucl-th/0203073.

[18] P. J. A. Bicudo, G. Krein and J. E. F. T. Ribeiro, Phys. Rev. C64 (2001) 025202 [hep-ph/0105289].

[19] A. Kvinikhidze and B. Blankleider, Phys. Rev. C60 (1999) 044003, 044004 nuclth/9901001, nucl-th/9901002.

[20] A. Kvinikhidze and B. Blankleider, in Nonperturbative Methods in Quantum Field Theory, edited by A. W. Schreiber, A. G. Williams, and A. W. Thomas (World Scientific, Singapore, 1998), p.284 [nucl-th/9806046]; Nucl. Phys. A670 (2000) 210c nuclth/9906017].

[21] V. Dmitrašinović, H.-J. Schulze, R. Tegen and R. H. Lemmer, Ann. Phys. (N.Y.) 238 (1995) 332.

[22] E. Nikolov, W. Broniowski, C. V. Christov, G. Ripka and K. Goeke, Nucl. Phys. A608 (1996) 411.

[23] M. Oertel, M. Buballa and J. Wambach, Nucl. Phys. A676 (2000) 247 [hep-ph/0001239].

[24] R. S. Plant and M. C. Birse, Nucl.Phys. A703 (2002) 717 [hep-ph/0007340].

[25] A. W. Thomas and G. Krein, Phys. Lett. B456 (1999) 5 nucl-th/9902013.

[26] A. W. Thomas and G. Krein, Phys. Lett. B481 (2000) 21 [nucl-th/0004008].

[27] L. Ya. Glozman, Phys. Lett. B459 (1999) 589 hep-ph/9904459.

[28] L. Ya. Glozman, Phys. Lett. B494 (2000) 58 [hep-ph/0004229].

[29] G. Ripka, Nucl. Phys. A683 (2001) 463 hep-ph/0003201]. 
[30] L. Ya. Glozman and D. O. Riska, Phys. Lett. B459 (1999) 49 hep-ph/9812224.

[31] B. Blankleider and A.N. Kvinikhidze, Phys. Rev. C62 039801 (2000) nucl-th/9912003.

[32] H. Asami, N. Ishii, W. Bentz and K. Yazaki, Phys. Rev. C51 (1995) 3388.

[33] M. Oettel, R. Alkofer and L. von Smekal, Eur. Phys. J. A8 (2000) 553 nucl-th/0006082.

[34] J. C. R. Bloch, C. D. Roberts and S. M. Schmidt, Phys. Rev. C61 (2000) 065207 nucl-th/9911068.

[35] A. N. Kvinikhidze and B. Blankleider, hep-th/0104053

[36] R. F. Wagenbrunn, S. Boffi, W. Klink, W. Plessas and M. Radici, Phys. Lett. B511 (2001) 33 [nucl-th/0010048. 